\title{
Comparison of microRNA profiles of human periodontal diseased and healthy gingival tissues
}

\author{
Yu-feng Xie ${ }^{1}$, Rong Shu ${ }^{1}$, Shao-yun Jiang ${ }^{1}$, Da-li Liu ${ }^{1}$, Xiu-li Zhang ${ }^{2}$ \\ ${ }^{I}$ Department of Periodontology, Shanghai Ninth People's Hospital Affiliated Shanghai Jiao Tong University School of \\ Medicine, Shanghai 200011, China; ${ }^{2}$ Shanghai Research Institute of Stomatology, Shanghai Ninth People's Hospital \\ Affiliated Shanghai Jiao Tong University School of Medicine, Shanghai 200011, China
}

MicroRNAs (miRNAs) have been demonstrated to play an important role in regulation of the immunoinflammatory response; however, the function of miRNAs in periodontal inflammation has not been investigated. The objective of this study was to explore the properties of miRNAs in periodontal inflammation by comparing miRNA profiles of inflamed and healthy gingival tissues. Gingival tissues were obtained from 10 periodontitis patients and 10 healthy subjects. After RNA extraction, miRNA profiles were analyzed by microarray, and expression levels of selected miRNAs were confirmed by real-time quantitative reverse transcription polymerase chain reaction (RT-PCR). Analyses using two computational methods, Targetscan and MicroRNA.org, were combined to identify common targets of these miRNAs. Finally, the individual miRNA expression levels of three toll-like receptor (TLR)-related miRNAs from inflamed and healthy gingival tissues were evaluated by RT-PCR. Ninety-one miRNAs were found to be upregulated and thirty-four downregulated over two-fold in inflamed gingival tissue compared with those in healthy gingival tissue. Twelve selected inflammatory-related miRNAs, hsa-miR-126*, hsa-miR-20a, hsa-miR-142-3p, hsa-miR-19a, hsa-let-7f, hsa-miR-203, hsa-miR-17, hsa-miR-223, hsa-miR-146b, hsa-miR-146a, hsa-miR-155, and hsa-miR-205 showed comparable expression levels by microarray and real-time quantitative RT-PCR analyses. In addition, the putative inflammation targets of these miRNAs were predicted, and three that were tested (hsa-miRNA-146a, hsa-miRNA-146b, and hsa-miRNA-155), showed significant differences between inflamed and healthy gingiva. This remarkable difference in miRNA profiles between periodontal diseased and healthy gingiva implicates a probable close relationship between miRNAs and periodontal inflammation. The data also suggest that the regulation of TLRs in periodontal inflammation may involve miRNA pathways.

Keywords: microRNA; microarray; periodontitis; gingival tissue

International Journal of Oral Science (2011) 3: 125-134. doi: 10.4248/IJOS 11046

\section{Introduction}

MicroRNAs (miRNAs) are small non-coding ribonucleic acid (RNA) molecules that are, on average, 22 nucleotides long. These molecules function as post-

*Correspondence: Rong Shu

Tel: 862123271699 5510; Fax: 862163135412

E-mail: shurong123@hotmail.com

Received 10 November 2010; Accepted 5 June 2011 transcriptional regulators that bind to complementary sequences in the three prime untranslated regions of target messenger RNA transcripts (mRNAs), usually resulting in gene silencing [1]. It has been suggested human genome may encode over 1000 miRNAs that are differentially expressed at high dynamic range in developmental stages, cell types, tissues, and diseases. In addition, by affecting gene regulation, miRNAs are likely to be involved in most biological processes, such as embryogenesis, differentiation, and carcinogenesis [2-4]. Recently, studies 
have begun to focus on the regulatory role of miRNAs in the inflammatory response [5-15]. Following polyribocytidylic acid or cytokine interferon- $\beta$ stimulation, miR155 was substantially up-regulated through activation of c-Jun N-terminal kinases pathway [9]. These studies of the roles of miRNAs in inflammatory processes can provide not only an in-depth understanding of immune homeostasis but also a novel therapeutic approach in the treatment of inflammatory disease.

Periodontitis is a complex chronic inflammatory disease of the periodontium, which results in a loss of connective tissue and bone support for the teeth. It is well known that bacteria-induced inflammatory responses are involved periodontal tissue damage, and the severity of chronic periodontitis is somewhat dependent on the dynamic equilibrium of interactions between the microbial challenge and the host immuno-inflammatory responses [16]. The local balance is tipped toward periodontal breakdown by excessive production of inflammatory cytokines and enzymes, such as interleukin (IL) $-1,-6$, tumor necrosis factor (TNF)- $\alpha$, prostaglandin E2, and matrix metalloproteinases. Other mediators, such as IL-4, IL-10, IL-1 receptor antagonist, and tissue inhibitors of metalloproteinases, can tip the balance toward periodontal stability. However, the exact regulatory mechanisms of the host immuno-inflammatory responses in chronic periodontitis remain obscure, and there are no reports regarding the role of miRNA in periodontal diseases. Therefore, in this study we used microarray analysis to compare global miRNA expression in gingival tissues between periodontitis patients and healthy subjects. Twelve differentially expressed inflammationrelated miRNAs were validated by real-time quantitative reverse transcription polymerase chain reaction (RTPCR) [17]. In addition, potential targets and functions of these inflammation-related miRNAs that may be involved in the regulation of local inflammation and connective tissue destruction were predicted and analyzed through computational methods. Moreover, three toll-like receptor (TLR)-related miRNAs from inflamed and healthy gingiva were evaluated by quantitative RT-PCR. Taken together, the objective of this study was to explore the properties of miRNAs in periodontal inflammation by comparing miRNA profiles of inflamed and healthy gingival tissues.

\section{Material and Methods}

\section{Subjects and sample collection}

This study population consisted of 20 subjects (10 periodontitis patients and 10 healthy control subjects), referred to Ninth People's Hospital, Shanghai Jiao Tong
University School of Medicine. Inflamed gingival tissues were collected from the active periodontal inflammation site of 10 chronic periodontitis subjects (4 males and 6 females, 28 to 63 years old) during periodontal flap surgery with inclusion criteria as follows: gingival index (GI) $>1$, at least 5 sites with probing depth $(\mathrm{PD}) \geqslant$ $5 \mathrm{~mm}$, clinical attachment loss $(\mathrm{CAL}) \geqslant 3 \mathrm{~mm}$, and extensive radiographic bone loss in the surgery quadrant while sampling. The periodontitis subjects had completed initial periodontal therapy before flap surgery. Healthy gingival tissues were collected from 10 periodontal healthy subjects ( 4 males and 6 females, 22 to 51 years old) during crown-lengthening procedures with $\mathrm{PD}<$ $3 \mathrm{~mm}, \mathrm{CAL}<1 \mathrm{~mm}$, and no radiographic evidence of alveolar bone loss in the entire dentition. All subjects were non-smokers, systemically healthy and did not receive any drug therapy within six months prior to tissue collection. Age and gender were matched in both groups. The purpose and procedures of the study were explained and informed consent obtained from each subject. The protocol followed in this study was approved by the ethics committee of Shanghai Ninth People's Hospital Affiliated Shanghai Jiao Tong University School of Medicine.

\section{RNA extraction}

Gingival tissue was immediately snap-frozen in liquid nitrogen and was ground to a fine powder in a mortar that was pre-cooled with liquid nitrogen to avoid RNA degradation. Total RNA was isolated using Trizol reagent (Invitrogen, CA, USA) according to the manufacturer's protocol and stored at $-80{ }^{\circ} \mathrm{C}$ until further use. RNA from either inflamed or healthy gingival tissues was pooled together and analyzed by commercial miRNA microarray (Kangchen Bio-Tech, Shanghai, China), while RNA from individual subjects was used for quantitative RT-PCR analysis.

\section{miRNA microarray analysis}

Briefly, miRNA in the RNA samples was labeled with fluorescent Hy3 using the miRCURYTM array-labeling kit (Exiqon, Denmark). The labeled miRNA was detected by hybridization to a miRNA microarray containing 1769 capture probes (miRCURYTM array microarray kit, v.11.0, Exiqon, Denmark) on Bioarray LifterSlip coverslip slides (Genetimes Technology, Shanghai, China). After being washed and dried by centrifugation, the slides were scanned using a microarray scanner Genepix 4000B with a $635 \mathrm{~nm}$ laser (Molecular Devices, CA, USA). The fluorescent density data in the images were analyzed using Genepix Pro 6.0 software (Molecular Devices, CA, USA) and are presented as the n-fold change (increase, I; decrease, D) of fluorescent density in inflamed gingiva 
(IG) after normalization to that in healthy gingiva (HG) as follows:

$$
\mathrm{I}=\frac{\mathrm{IG}}{\mathrm{HG}} \quad \mathrm{D}=-\frac{\mathrm{HG}}{\mathrm{IG}}
$$

The median normalization method was used to obtain "Normalized Data":

$$
\text { Normalized Data }=\frac{\text { Foreground }- \text { Background }}{\text { median }}
$$

in which the median is equal to $50 \%$ of the microRNA intensity larger than 50 in all samples following background correction.

\section{Real-time quantitative RT-PCR}

Total RNA was treated with RNase-free DNase (Epicentre, Madison, USA) and then reverse transcribed to create cDNA using the MMLV Reverse Transcriptase First Strand cDNA Synthesis Kit (Epicentre, Madison, USA). The 54-mer RT primers were designed to include a 48-mer stem-loop fragment, 5'-GTCGTATCCAGTGCGTGTCGTGGAGTCGGCAATTGCACTGGATACGAC-3', to six oligonucleotides of the $3^{\prime}$ portion of the target miRNA. Each reaction mixture of the reverse transcription contained $2 \mu \mathrm{g}$ of DNase-treated total RNA, $50 \mathrm{nmol} \cdot \mathrm{L}^{-1} \mathrm{RT}$ primer, $1 \times$ reaction buffer, $0.25 \mathrm{mmol} \cdot \mathrm{L}^{-1}$ of each dNTP, $200 \mathrm{U}$ MMLV Reverse Transcriptase, $20 \mathrm{U}^{\text {ScriptGuard }}{ }^{\mathrm{TM}}$ RNase Inhibitor, and nuclease-free water (Epicentre, Madison, USA) to a total volume of $20 \mu \mathrm{L}$. The reaction was performed at $16{ }^{\circ} \mathrm{C}$ for $30 \mathrm{~min}$, $42{ }^{\circ} \mathrm{C}$ for $30 \mathrm{~min}$, followed by $85^{\circ} \mathrm{C}$ for $5 \mathrm{~min}$ in an
Applied Biosystems 9700 Thermocycler (Applied Biosystems, USA). RNA samples from the 10 healthy and 10 periodontitis subjects were pooled for this analysis. We selected 12 miRNAs that were differently expressed in inflamed and healthy gingival tissues for further validation of the data obtained from microarray analysis using real-time quantitative RT-PCR.

RT-PCR was performed following a standard SYBR Green PCR protocol using RT SYBR ${ }^{\circledR}$ Green qPCR Master Mixes (PA-112, SAbiosciences, Qiagen) on an Applied Biosystems 7900HT Sequence Detection System (Applied Biosystems, USA). The U6 small nuclear RNA (NR_003027) was used as an internal control. Each reaction contained $5 \mu \mathrm{L}$ of RT $\mathrm{SYBR}^{\circledR}$ Green qPCR Master Mixes, $1.5 \mu \mathrm{mol} \cdot \mathrm{L}^{-1}$ of a gene-specific forward PCR primer that bound to the 5'-portions of target miRNA, $0.7 \mu \mathrm{mol} \cdot \mathrm{L}^{-1}$ of a universal reverse PCR primer that bound to the sequence located in the stem-loop structure, and nuclease-free water to a total volume of $10 \mu \mathrm{L}$. Triplicate reactions were performed at $95{ }^{\circ} \mathrm{C}$ for $10 \mathrm{~min}$, followed by 40 cycles of $95{ }^{\circ} \mathrm{C}$ for $15 \mathrm{~s}, 60{ }^{\circ} \mathrm{C}$ for $15 \mathrm{~s}, 72{ }^{\circ} \mathrm{C}$ for $15 \mathrm{~s}$, and $55{ }^{\circ} \mathrm{C}$ for $15 \mathrm{~s}$. All reactions were repeated twice. The sequences of target mature miRNAs and specific forward PCR primers are listed in Table 1. The universal reverse PCR primer sequence is 5'-CAGTGCGTGTCGTGGAGT-3', and the primer pairs used for the U6 small nuclear RNA are U6-forward 5'-GCTTCGGCAGCACATATACTAAAAT-3' and U6reverse 5'-CGCTTCACGAATTTGCGTGTCAT-3'.

The relative expression of miRNA as compared with U6 was calculated using the $2^{-\Delta \mathrm{CT}}$ method [18], where

\begin{tabular}{|c|c|c|}
\hline Target miRNA & Mature miRNA sequence $^{\mathrm{a}}$ & Specific forward PCR primer ${ }^{b}$ \\
\hline hsa-miR-126*c & CATTATTACTTTTGGTACGCG & GGGGCATTATTACTTTTGG \\
\hline hsa-miR-20a & TAAAGTGCTTATAGTGCAGGTAG & GGGGTAAAGTGCTTATAGTGC \\
\hline hsa-miR-142-3p & TGTAGTGTTTCCTACTTTATGGA & GGGGTGTAGTGTTTCCTACT \\
\hline hsa-miR-19a & TGTGCAAATCTATGCAAAACTGA & GGGTGTGCAAATCTATGCA \\
\hline hsa-let-7f & TGAGGTAGTAGATTGTATAGTT & GGGGTGAGGTAGTAGATTGT \\
\hline hsa-miR-203 & GTGAAATGTTTAGGACCACTAG & GGGGTGAAATGTTTAGGAC \\
\hline hsa-miR-17 & CAAAGTGCTTACAGTGCAGGTAG & CCCAAAGTGCTTACAGTGC \\
\hline hsa-miR-223 & TGTCAGTTTGTCAAATACCCCA & GGGGTGTCAGTTTGTCAAAT \\
\hline hsa-miR-146b-5p & TGAGAACTGAATTCCATAGGCT & GGGTGAGAACTGAATTCCA \\
\hline hsa-miR-146a & TGAGAACTGAATTCCATGGGTT & GGGTGAGAACTGAATTCCA \\
\hline hsa-miR-155 & TTAATGCTAATCGTGATAGGGGT & GGGTTAATGCTAATCGTGAT \\
\hline hsa-miR-205 & TCCTTCATTCCACCGGAGTCTG & GGTCCTTCATTCCACCG \\
\hline
\end{tabular}

Table 1 Oligonucleotide sequences of target mature miRNAs and specific forward PCR primers ( $5^{\prime}$ to $\left.3^{\prime}\right)$

${ }^{a}$ Mature miRNAs shown were obtained from www.mirbase.org. Underlined letters represent the complementary sequences within the 3'-portion of the stem-loop RT primers.

${ }^{\mathrm{b}}$ Italic letters represent the sequences that specifically bind the 5'-portion of the cDNA from the target mature miRNA.

${ }^{\mathrm{c}}$ The asterisks $(*)$ refer to different products from the same pre-miRNA. 


$$
\Delta \mathrm{CT}=\Delta \mathrm{CT}_{\text {sample } 2}-\Delta \mathrm{CT}_{\text {sample } 1}
$$

$\Delta \mathrm{CT}_{\text {sample2 }}$ and $\Delta \mathrm{CT}_{\text {sample1 }}$ are the $\mathrm{Ct}$ values for the sample 2 and sample 1 , respectively, normalized to an endogenous housekeeping gene.

\section{Computational predication of miRNA targets}

To further analyze the functions of miRNAs identified, we used two computational approaches, MicroRNA.org (www.microrna.org) and targetscan (www.targetscan. org), to predict common targets of the miRNAs and differentially expressed miRNAs corresponding to putative genes [19-20]. The mirSVR algorithm tool from MicroRNA.org was used to evaluate many features of the miRNA targets identified, including both secondary structure-based accessibility of the target site and conservation without introducing a large number of spurious predictions [20]. Targets that were predicted by both modules and acceptable mirSVR down-regulation scores were selected for additional studies.

\section{Statistical analysis}

Statistical analysis was performed using an unpaired Student's $t$ test. Differences were considered significant when the probability value was less than $5 \%(P<0.05)$.

\section{Results}

miRNA profiles of gingival tissues from periodontitis patients and healthy subjects

As listed in Table 2, 91 miRNAs were upregulated as compared with controls, and five of these miRNAs showed more than a five-fold change. In addition, there were 85 miRNAs upregulated two-to-five fold, and 34 miRNAs down-regulated two-to-five fold.

Table 2 Differential expression of miRNAs in inflamed and healthy gingival tissues ${ }^{\mathrm{a}}$

\begin{tabular}{|c|c|c|}
\hline Range of fold change ${ }^{b}$ & Number & miRNA \\
\hline $5-10(\mathrm{I})$ & 5 & hsa-miR-126*c , hsa-miR-190, hsa-miR-20a, hsa-miR-32, hsa-miR-362-3p \\
\hline \multirow[t]{15}{*}{$2-5(\mathrm{I})$} & \multirow[t]{15}{*}{86} & hsa-let-7a, hsa-let-7f, hsa-let-7g*, hsa-miR-101, hsa-miR-101*, hsa-miR-105, hsa-miR-106a, \\
\hline & & hsa-miR-10b, hsa-miR-1207-5p, hsa-miR-122*, hsa-miR-1225-3p, hsa-miR-1257, \\
\hline & & hsa-miR-1259, hsa-miR-126, hsa-miR-1266, hsa-miR-1297, hsa-miR-130a, hsa-miR-130a*, \\
\hline & & hsa-miR-130b*, hsa-miR-132*, hsa-miR-136*, hsa-miR-140-5p, hsa-miR-141*, \\
\hline & & hsa-miR-142-3p, hsa-miR-144*, hsa-miR-17, hsa-miR-181c, hsa-miR-18b, hsa-miR-194, \\
\hline & & hsa-miR-195, hsa-miR-19a, hsa-miR-203, hsa-miR-20a*, hsa-miR-218, hsa-miR-219-5p, \\
\hline & & hsa-miR-221*, hsa-miR-26b, hsa-miR-27a, hsa-miR-27b, hsa-miR-29b-2*, hsa-miR-301a, \\
\hline & & hsa-miR-302b, hsa-miR-302d, hsa-miR-30b, hsa-miR-30e, hsa-miR-30e*, hsa-miR-31*, \\
\hline & & hsa-miR-335, hsa-miR-338-3p, hsa-miR-33a, hsa-miR-34c-5p, hsa-miR-363*, \\
\hline & & hsa-miR-369-5p, hsa-miR-374a, hsa-miR-374a*, hsa-miR-374b*, hsa-miR-379, hsa-miR-411, \\
\hline & & hsa-miR-411*, hsa-miR-424, hsa-miR-450b-5p, hsa-miR-452*, hsa-miR-455-3p, \\
\hline & & hsa-miR-488, hsa-miR-507, hsa-miR-514, hsa-miR-518c, hsa-miR-520a-5p, hsa-miR-520b, \\
\hline & & hsa-miR-523, hsa-miR-524-5p, hsa-miR-532-3p, hsa-miR-582-5p, hsa-miR-587, \\
\hline & & hsa-miR-590-5p, hsa-miR-592, hsa-miR-593*, hsa-miR-609, hsa-miR-644, hsa-miR-648, \\
\hline & & hsa-miR-769-3p, hsa-miR-886-5p, hsa-miR-889, hsa-miR-9*, hsa-miR-922, hsa-miR-95 \\
\hline \multirow[t]{7}{*}{$-5--2(\mathrm{D})$} & \multirow[t]{7}{*}{34} & hsa-miR-1181, hsa-miR-1205, hsa-miR-1274b, hsa-miR-1275, hsa-miR-1277, \\
\hline & & hsa-miR-138-2*, hsa-miR-182*, hsa-miR-187, hsa-miR-188-3p, hsa-miR-200a*, \\
\hline & & hsa-miR-204, hsa-miR-208b, hsa-miR-214*, hsa-miR-299-3p, hsa-miR-302a, hsa-miR-302c, \\
\hline & & hsa-miR-30c- ${ }^{*}$, hsa-miR-323-5p, hsa-miR-33b, hsa-miR-431, hsa-miR-451, \\
\hline & & hsa-miR-485-5p, hsa-miR-487a, hsa-miR-488*, hsa-miR-502-5p, hsa-miR-513c, \\
\hline & & hsa-miR-542-5p, hsa-miR-543, hsa-miR-548e, hsa-miR-571, hsa-miR-589*, hsa-miR-597, \\
\hline & & hsa-miR-605, hsa-miR-92a-2* \\
\hline
\end{tabular}

${ }^{\mathrm{a}}$ Data were calculated based on fluorescent density quantitated from microarray images analyzed using Genepix Pro 6.0 software.

${ }^{b}$ Fold change (increase, I; decrease, D) represent that of fluorescent density in inflamed gingiva (IG) after normalization to that in healthy gingiva $(\mathrm{HG})$ as follows: $\mathrm{I}=\mathrm{IG} / \mathrm{HG} ; \mathrm{D}=-\mathrm{HG} / \mathrm{IG}$.

${ }^{\mathrm{c}}$ The asterisks $(*)$ refer to different products from the same pre-miRNA. 
Real-time quantitative RT-PCR for miRNA precursors

Of the 12 miRNAs examined, seven showed an increased expression of more than two-fold, and 5 miRNAs showed either an increased or decreased expression of 1.5- to two-fold in inflamed gingival tissue as compared to controls. Specifically, the expression of hsa-miR-126*, hsa-miR-20a, hsa-miR-142-3p, hsa-miR-19a, hsa-let-7f, hsa-miR-203, hsa-miR-17, hsa-miR-223, hsa-miR-146b, and hsa-miR-146a increased and the expression of hsa-miR-155 and hsa-miR-205 decreased in inflamed periodontal tissues relative to healthy tissues.

Primers containing a stem-loop were designed for reverse transcription cDNA synthesis, and gene-specific PCR primers were used for real-time quantitative PCR. As shown in Figure 1, all 12 of the miRNAs tested showed comparable expression levels by microarray and real-time PCR analysis, further confirming that these inflammation-related miRNAs may participate in periodontal infection.

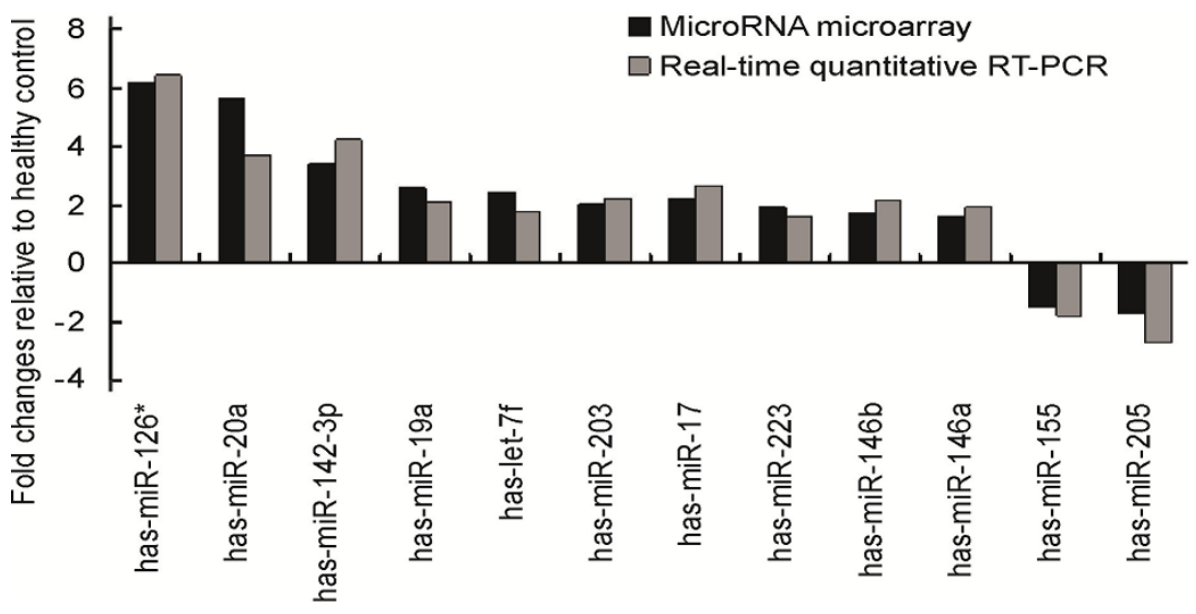

Figure 1 Relative expression levels of 12 selected miRNAs in periodontal inflamed and healthy gingival tissues analyzed by microRNA microarray and real-time quantitative RT-PCR. The 12 inflammation-related miRNAs are listed on the horizontal ordinate. The vertical ordinate refers to fold changes of the relative expression levels between inflamed gingiva and healthy gingiva. Black bars refer to microarray results, and grey bars refer to the real-time quantitative RT-PCR results.

\section{Putative miRNA target gene prediction}

Because the functions of most miRNAs are unknown, we used MicroRNA.org (August 2010 Release, www. microrna.org) and targetscan (www.targetscan.org) to identify potential common targets of the miRNAs identified.

As expected, miRNA target prediction showed that these 12 selected miRNAs functioned in inflammatory processes, including inflammation-related signaling (hsamiR-146a, hsa-miR-146b, hsa-miR-19a, hsa-miR-20a, hsa-miR-142-3p, hsa-miR-155 and hsa-miR-203), leukocyte adherence and vessel hyperplasty in inflammation (hsa-miR-126*), cell proliferation and differentiation of fibroblasts (hsa-miR-205, hsa-miR-223 and hsa-miR-155), apoptosis (hsa-let-7f, hsa-miR-19a, hsa-miR-20a, hsamiR-203 and hsa-miR-223), and bone resorption caused by inflammation (hsa-miR-17 and hsa-miR-20a). These results suggest that one target can be either upregulated or downregulated by a group of miRNAs that share a common regulatory mechanism and function as a biological network. The predictive inflammatory response targets and mirSVR downregulation scores of these miRNAs are listed in Table 3.

\section{Expression of TLR-related miRNAs in individual tissues}

According to the target gene prediction algorithm, three microRNAs, hsa-miR-146a, hsa-miR-146b, and hsa-miR-155, have a functional role in TLR-related regulation in inflammatory diseases. The expression levels of these three miRNAs in seven individual samples from each group were further analyzed by quantitative RT-PCR. As expected, hsa-miR-146a and hsa-miR-146b expression levels were significantly higher in inflamed tissues as compared with healthy tissues, while hsamiR-155 expression was significantly lower in inflamed tissues as compared with healthy controls $(P<0.05)$, as shown in Figure 2. 
Table 3 Putative inflammation-related targets of selected miRNAs

\begin{tabular}{|c|c|}
\hline miRNA & Putative targets ${ }^{\mathrm{a}, \mathrm{b}}$ \\
\hline hsa-miR-126*c & VCAM- ${ }^{\mathrm{d}}$, SPRED- ${ }^{\mathrm{e}}$ \\
\hline hsa-miR-20a & $\begin{array}{l}\left.\text { RANKL (TNFSF-11) (-1.041 2 } 2^{f}\right) \text {, STAT-3 (-0.740 1), FGF-7 (-0.675 6), BCL-11B (-0.670 6), MAPK-9 (-0.307 5), } \\
\text { BMPR-2 (-0.162 7) }\end{array}$ \\
\hline hsa-miR-142-3p & STAM (-1.296 1), TGFBR-1 (-0.519 3), IRAK1 (-0.185 1) \\
\hline hsa-miR-19a & $\begin{array}{l}\text { SOCS3 (-1.210 2), COL-1A2 (-1.144 2), BCL-2-L11 (-0.554 0), IL-1R1 (-0.465 2), BMPR-2 (-0.198 1), } \\
\text { SOCS1 (-0.103 2) }\end{array}$ \\
\hline hsa-let-7f & COL-1A2 (-1.272 7), TGFBR-1 (-0.446 5), BCL-2-L1 (-0.237 3) \\
\hline hsa-miR-203 & SOCS-3 (-1.268 2), BCL-2-L2 (-0.994 4), TLR-4 (-0.885 7), BCL-11B (-0.482 5), BCL-2 (-0.124 3) \\
\hline hsa-miR-17 & RANKL (TNFSF-11) (-1.038 5), BMPR-2 (-0.162 7), STAT-3 (-0.162 7) \\
\hline hsa-miR-223 & FGFR-2 (-0.806 0), NFIB (-0.501 0), FGF-2 (-0.328 2) \\
\hline hsa-miR-146b & TRAF-6 (-1.121 6), IRAK-1 (-0.882 1), TLR4 (-0.123 4) \\
\hline hsa-miR-146a & TRAF-6 (-1.121 6), IRAK-1 (-0.882 1), TLR4 (-0.123 4) \\
\hline hsa-miR-155 & SOCS-5 (-1.161 8), SOCS-1 (-1.147 2), FGF-7 (-0.935 0), IKK $\beta$, IKK $\varepsilon$, FADD, Ripk1 ${ }^{\mathrm{g}}$ \\
\hline hsa-miR-205 & VEGFA (-0.904 9), NFIB (-0.752 8), IL-1R1 (-0.660 2), BCL-2 (-0.300 0), FGFR-1 (-0.197 3), FGF1 (-0.179 6) \\
\hline
\end{tabular}

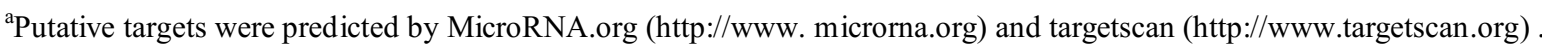

bAbbreviations: BCL-11B: B-cell lymphoma-11B; BCL-2: B-cell lymphoma-2; BCL-2-L1: BCL2-like-1; BCL-2-L2: BCL2-like 2; BCL-2-L11: BCL2-like 11; BMPR-2: bone morphogenetic protein receptor, type II; COL-1A2: collagen, type I, alpha 2; FADD: Fas-associated death domain; FGF-1: fibroblast growth factor-1; FGF-2: fibroblast growth factor 2 (basic); FGF-7: fibroblast growth

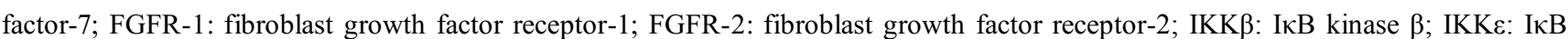
kinase $\beta$; IL1-R1: interleukin-1 receptor, type I; IRAK-1: interleukin-1 receptor-associated kinase-1; MAPK-9: mitogen-activated protein kinase-9; NFIB: nuclear factor I/B; RANKL: receptor activator of NF- $\mathrm{B}$ ligand; Ripk1: receptor-interacting serine/threonineprotein kinase 1; SOCS-1: suppressor of cytokine signaling 1; SOCS-3: suppressor of cytokine signaling-3; SOCS-5: suppressor of cytokine signaling-5; SPRED1: sprouty-related EVH1 domain containing 1; STAM: signal transducing adaptor molecule; STAT-3: signal transducer and activator of transcription-3; TGFBR-1: transforming growth factor, beta receptor I; TLR-4: toll-like receptor-4; TNFSF-11: tumor necrosis factor (ligand) superfamily, member-11; TRAF-6: TNF receptor-associated factor-6; VEGFA: vascular endothelial growth factor A; VCAM-1: vascular cell adhesion molecule 1.

${ }^{\mathrm{c}}$ The asterisks $(*)$ refer to different products from the same pre-miRNA.

${ }^{\mathrm{d}}$ refer to [15].

e refer to [35].

${ }^{\mathrm{f}}$ mirSVR scores by www.microrna.org.

${ }^{\mathrm{g}}$ refer to [33].

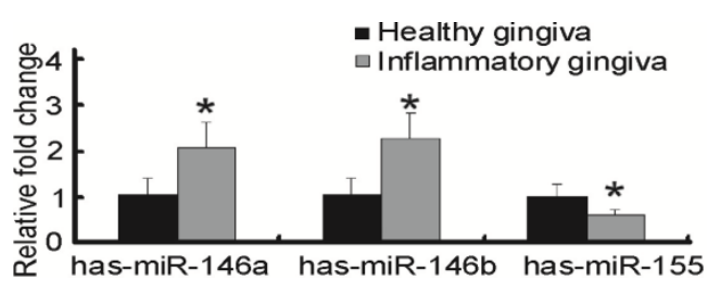

Figure 2 Relative fold changes of hsa-miR-146a, hsamiR-146b, and hsa-miR-155 in periodontal inflamed and healthy gingiva evaluated by quantitative RT-PCR. hsa-miR-146a and hsa-miR-146b were upregulated, while hsa-miR-155 was downregulated in periodontal inflamed gingival tissue compared with those in healthy gingival tissue. Values and error bars represent the means and SDs of seven individual subjects, ${ }^{*} P<0.05$ versus healthy controls.

\section{Discussion}

Periodontal inflammation is initiated by a set of consecutive events, including an immune response, cell apoptosis, cell proliferation, differentiation, and inflammatory cell adhesion and migration. According to sampled survey data from the third national oral health survey, the prevalence and severity of periodontal disease is increased during aging. The rate of complete periodontal health was only $14.5 \%$ in 35 -to-45-year old people. The inflammatory gingival tissues analyzed in our study were collected from 10 periodontitis subjects ( 4 males and 6 females who were 28 to 63 years old with an average age of 40.6 years), and the results of this study reflect an accurate assessment of miRNA expression levels in healthy and inflamed gingival tissues. 
In this study, we compared miRNA profiles of inflamed gingival tissues with healthy gingival tissues and found 125 microRNAs to be either upregulated or downregulated over two-fold. Among the 91 upregulated miRNAs, 5 miRNAs were upregulated more than fivefold in inflamed periodontal tissues (Table 2). These differentially expressed miRNAs were originally found to be expressed in a variety of organs and tissues, including airway epithelial cells (hsa-miR-126) and skin (hsa-miR-203), and have been implicated to have roles in other diseases and biological processes [7, 21].

Abnormal miRNA expression may lead to more rapid disease progression. Previous studies have implicated miRNAs in human diseases such as cancer [22-24], developmental abnormalities [25], muscular diseases [26] and cardiovascular disorders [27-28]. The importance of miRNA has also been recognized in several inflammatory diseases [5-7, 10-11]; however, the potential role of miRNAs in periodontal inflammatory regulation has remained unknown. This study is the first to analyze miRNA profiles from inflamed and healthy periodontal tissues and to consequently reveal the disparities in miRNA expression levels between diseased and healthy subjects. These data provide potential candidates for further investigation of how miRNAs contribute to disease mechanisms and for possible therapeutic treatment by specifically targeting the host immuno-inflammatory response in periodontal diseases.

Primers containing a stem-loop were designed for cDNA synthesis, and gene-specific PCR primers were used for real-time quantitative PCR. This strategy was more efficient and specific than conventional methods used for real-time quantitative RT-PCR for miRNA amplification described in previous reports and therefore was employed in this study [29]. We selected 12 inflammation-related miRNAs to validate their expression level with real-time quantitative RT-PCR (Figure 1). The quantitative RT-PCR data correlated well with microarray analysis and demonstrated the reliability of microarray assay. mRNA target prediction of these miRNAs showed that they may function in inflammation-related signaling, leukocyte adherence, vessel hyperplasty in inflammation, cell proliferation and differentiation of fibroblasts, and bone resorption caused by inflammation (Table 3).

Based on the target gene prediction results, we selected three microRNAs that are functionally associated with TLR-related regulation in inflammatory diseases, hsa-miR-146a, hsa-miR-146b, and hsa-miR-155, for expression level analysis in individual samples. The expression of hsa-miR-146a and hsa-miR-146b were both significantly higher, while the expression of hsa-
miR-155 was significantly lower in inflamed tissues as compared with healthy control tissues (Figure 2). These data suggest that these microRNAs may play a role in periodontal inflammation via regulation of or interaction with TLR-related pathways.

hsa-miR-146a is located in the second exon of the LOC285628 gene on human chromosome 5, and hsamiR-146b resides on chromosome 10 . These two miRNAs have been confirmed to be involved in inflammatory diseases such as rheumatoid arthritis and psoriasis [5-7, 12]. Taganov et al. [14] found that hsamiR-146a/b expression could be induced by many proinflammatory stimuli including TLR ligands (TLR2, TLR4, and TLR5) and cytokines, such as TNF- $\alpha$ and IL-1 $\beta$, in a nuclear factor $\mathrm{kB}(\mathrm{NF}-\mathrm{kB})$-dependent manner. The direct targets of hsa-miR-146a include IL-1 receptor associated kinase (IRAK1) and TNF receptor-associated factor-6 (TRAF6), which are key components in the TLR4 signaling pathway. However, increased hsa-miR146a expression negatively regulated the release of IL-8 and regulated upon activation normal $\mathrm{T}$ cell expressed and secreted (RANTES), and inhibitors of hsa-miR-146 resulted in increased IL-8 and RANTES [13]. In addition, Hung et al. [30] found that immortalized periodontal ligament (I-PDL) cells that expressed exogenous hsamiR-146a showed higher mRNA levels of alkaline phosphatase, osteocalcin, and osteopontin, as well as an attenuation of NF- $\kappa \mathrm{B}$ activity, suggesting a possible pathway of hsa-miR-146a in the regulation of periodontal cells. Nahid et al. [31] investigated the expression of miRNAs in maxilla (periodontium) isolated from ApoE (-/-) mice infected with periodontal pathogens and found that miR-146a expression was negatively correlated with TNF- $\alpha$ secretion in vitro, causing reduced levels of IRAK-1 and TRAF6 adaptor kinases. Thus, hsa-miR-146 may be involved in a negative feedback mechanism to regulate TLR signaling in response to bacterial products. In this study, the expression of hsa-miR-146a/b increased in inflamed gingival tissues as compared with healthy gingival tissues, suggesting that increased hsa-miR-146 expression may protect gingival tissue from suffering excess inflammatory damage.

Mature hsa-miR-155 is generated from a precursor form known as BIC RNA, which is encoded by the bic gene [32], and is known to be linked to a number of human neoplastic disorders and inflammatory diseases. Previous studies have reported that the expression of miR-155 was upregulated in splenocytes, monocytes and macrophages of mice treated with LPS or lipoprotein, which stimulate TLR-4 or TLR-2, respectively [9, 14, 33]. In contrast to miR-146 expression, which was primarily regulated by bacterial envelope products, miR-155 was 
shown to be regulated by viral- and bacterial-derived nucleotides including poly(I:C) and CpG via TLR-3 and TLR-9, respectively $[9,14]$. Interestingly, miR-155 may be involved in both positive and negative regulation of target intercellular mediators, such as I $\mathrm{B}$ kinase $\beta$, I $\mathrm{B}$ kinase $\varepsilon$, Fas-associated death domain (FADD), receptor interacting serine-threonine kinase 1 (Ripk1), and other NF-kB signaling proteins [33]. By enhancing TNF- $\alpha$ translation, miR-155 may regulate the LPS/TNF- $\alpha$ pathway during host immune responses.

Stanczyk et al. [11] reported upregulation of miR-155 resulting from stimulation with TLR ligands that appeared to be a common response of fibroblasts and monocytes in rheumatoid arthritis patients. In contrast, Nakasa et al. [8] observed strong induction of miR-146a following $\mathrm{TNF} \alpha$ stimulation and did not observe upregulation of miR-155 in rheumatoid arthritis synovial fibroblasts. In this study, hsa-miR-155 expression decreased in inflamed gingival tissue, which is inconsistent with previous rheumatoid arthritis study. This contradictory result may be due to differences in the tissue and cell types studied, especially considering that gingiva is complex tissue composed of many cell types, including gingival fibroblasts, epithelial cells, nerves and blood vessels, which adds significant complexity to the regulation progress. Future studies are necessary to determine the cell components and mechanisms that led to these changes in miRNA expression levels due to periodontal inflammation.

Apart from the three TLR-related miRNAs, other inflammation-related miRNAs may also play important roles in the regulation of periodontal inflammation. As shown in Table 3, their targets include suppressor of cytokine signaling (SOCS) (hsa-miR-155, hsa-miR-19a and hsa-miR-203), vascular cell adhesion molecule (VCAM)-1 (hsa-miR-126), sprouty-related EVH1 domain containing 1 (SPRED-1) (hsa-miR-126), fibroblast growth factors (FGFs) and their receptors (hsa-miR-20a, hsa-miR-223, hsa-miR-205 and hsa-miR-155), B-cell lymphoma (Bcl) family members (hsa-miR-19a, hsamiR-20a, hsa-let-7f, hsa-miR-203 and hsa-miR-205), and receptor activator of $\mathrm{NF}-\mathrm{\kappa B}$ ligand (RANKL) (hsa-miR-17 and hsa-miR-20a). These targets have been reported to be involved in a wide range of inflammatory pathways. For example, SOCS serves as a negative regulator of the STAT3 pathway [34]. VCAM-1 is a key regulator in leukocyte trafficking to sites of inflammation [15], and SPRED-1 is associated with vessel formation [35]. FGFs and fibroblast growth factor receptors (FGFRs) are key players in the processes of fibroblast proliferation and differentiation of gingival tissues [36-38]. Bcl family members act as anti- or pro-apoptotic regulators, which are involved in a wide variety of cellular activities [39-40]. RANKL is a critical factor required in the processing of alveolar bone resorption [41]. The precise effect of these miRNAs on their target genes remains to be investigated.

\section{Conclusions}

In conclusion, we explored miRNA expression patterns in inflamed gingival tissue. Using microarray assay analysis, we examined miRNA expression in inflamed and healthy gingival tissues, and 125 miRNAs were identified as differentially expressed more than two-fold. To elucidate the role of these miRNAs in the inflammatory response, we selected 12 miRNAs whose expression levels significantly changed in inflamed gingival tissue as compared with healthy tissue and conformed their expression pattern in the inflamed gingival tissue by real-time quantitative RT-PCR. Although this study did not determine the location and the putative target levels of these miRNAs in periodontal tissues and cell components, the present data provide candidates for further analysis of miRNAs in periodontal infection and for the development of potential biomarkers and therapeutic targets for periodontitis.

In future studies, we will investigate the mechanisms that lead to these interesting changes in miRNA expression and continue to explore the roles of miRNAs in periodontal inflammation.

\section{Acknowledgements}

This work was supported by the Science and Technology Commission of Shanghai (Project No. 08JC 1414600), the Doctoral Innovation Fund of Shanghai Jiao Tong University School of Medicine (Project No. BXJ201030) and the Shanghai Health Bureau Science Fund for Young Scholars (Project No. 2010165).

\section{Competing interests}

No competing financial interests or personal relationships exist.

\section{References}

1 Bartel DP. MicroRNAs: genomics, biogenesis, mechanism, and function. Cell 2004; 116: 281-297.

2 Bi Y, Liu G, Yang R. MicroRNAs: novel regulators during the immune response. J Cell Physiol 2009; 218: 467-472.

3 Friedman RC, Farh KK, Burge CB, Bartel DP. Most mammalian mRNAs are conserved targets of microRNAs. 
Genome Res 2009; 19: 92-105.

4 Osada H, Takahashi T. MicroRNAs in biological processes and carcinogenesis. Carcinogenesis 2007; 28: 2-12.

5 Sonkoly E, Ståhle M, Pivarcsi A. MicroRNAs and immunity: novel players in the regulation of normal immune function and inflammation. Semin Cancer Biol 2008; 18: 131-140.

6 Sonkoly E, Ståhle M, Pivarcsi A. MicroRNAs: novel regulators in skin inflammation. Clin Exp Dermatol 2008; 33: $312-315$.

7 Sonkoly E, Wei T, Janson PC, et al. MicroRNAs: novel regulators involved in the pathogenesis of Psoriasis? PLoS ONE 2007; 2: e610.

8 Nakasa T, Miyaki S, Okubo A, et al. Expression of microRNA-146 in rheumatoid arthritis synovial tissue. Arthritis Rheum 2008; 58: 1284-1292.

9 O'Connell RM, Taganov KD, Boldin MP, Cheng G, Baltimore D. MicroRNA-155 is induced during the macrophage inflammatory response. Proc Natl Acad Sci USA 2007; 104: 1604-1609.

10 Lindsay MA. MicroRNAs and the immune response. Trends Immunol 2008; 29: 343-351.

11 Stanczyk J, Pedrioli DM, Brentano F, et al. Altered expression of MicroRNA in synovial fibroblasts and synovial tissue in rheumatoid arthritis. Arthritis Rheum 2008, 58: 1001-1009.

12 Pauley KM, Satoh M, Chan AL, et al. Upregulated miR-146a expression in peripheral blood mononuclear cells from rheumatoid arthritis patients. Arthritis Res Ther 2008; 10: R101.

13 Perry MM, Moschos SA, Williams AE, et al. Rapid changes in microRNA-146a expression negatively regulate the IL-1 $\beta$ induced inflammatory response in human lung alveolar epithelial cells. J Immunol 2008; 180: 5689-5698.

14 Taganov KD, Boldin MP, Chang KJ, Baltimore D. NFkappaB-dependent induction of microRNAmiR-146, an inhibitor targeted to signaling proteins of innate immune responses. Proc Natl Acad Sci U S A 2006; 103: 1248112486.

15 Harris TA, Yamakuchi M, Ferlito M, Mendell JT, Lowenstein CJ. MicroRNA-126 regulates endothelial expression of vascular cell adhesion molecule 1. Proc Natl Acad Sci US A 2008; 105: 1516-1521.

16 Kornman KS. Mapping the pathogenesis of periodontitis: a new look. J Periodontol 2008; 79: 1560-1568.

17 Chen Y, Gelfond JA, McManus LM, Shireman PK. Reproducibility of quantitative RT-PCR array in miRNA expression profiling and comparison with microarray analysis. BMC Genomics 2009; 10: 407-415.

18 Livak KJ, Schmittgen TD. Analysis of relative gene expression data using real-time quantitative PCR and the 2(-Delta Delta C(T)) method. Methods 2001; 25: 402-408.

19 Friedman JM, Jones PA. MicroRNAs: critical mediators of differentiation, development and disease. Swiss Med Wkly 2009; 139: 466-472.

20 Betel D, Koppal A, Agius P, Sander C, Leslie C. Comprehensive modeling of microRNA targets predicts functional non-conserved and non-canonical sites. Genome Biol 2010; 11: R90.

21 Oglesby IK, Bray IM, Chotirmall SH, et al. miR-126 is downregulated in cystic fibrosis airway epithelial cells and regulates TOM1 expression. J Immunol 2010; 184: 17021709.

$22 \mathrm{Lu} \mathrm{J}$, Getz G, Miska EA, et al. MicroRNA expression profiles classify human cancers. Nature 2005; 435: 834 838.

23 Calin GA, Croce CM. MicroRNA signatures in human cancers. Nat Rev Cancer 2006; 6: 857-866.

24 Croce CM, Calin GA. miRNAs, cancer, and stem cell division. Cell 2005; 122: 6-7.

25 Kloosterman WP, Lagendijk AK, Ketting RF, Moulton JD, Plasterk RH. Targeted inhibition of miRNA maturation with morpholinos reveals a role for miR-375 in pancreatic islet development. PLoS Biol 2007; 5: e203.

26 Eisenberg I, Eran A, Nishino I, et al. Distinctive patterns of microRNA expression in primary muscular disorders. Proc Natl Acad Sci U S A 2007; 104: 17016-17021.

27 Ikeda S, Kong SW, Lu J, et al. Altered microRNA expression in human heart disease. Physiol Genomics 2007; 31: 367-373.

28 Cheng Y, Ji R, Yue J, et al. MicroRNAs are aberrantly expressed in hypertrophic heart: do they play a role in cardiac hypertrophy? Am J Pathol 2007; 170: 1831-1840.

29 Chen C, Ridzon DA, Broomer AJ, et al. Real-time quantification of microRNAs stem-loop RT-PCR. Nucleic Acids Res 2005; 33: e179.

30 Hung PS, Chen FC, Kuang SH, et al. miR-146a induces differentiation of periodontal ligament cells. $J$ Dent Res 2010; 89: 252-257.

31 Nahid MA, Rivera M, Lucas A, Chan EK, Kesavalu L. Polymicrobial infection with periodontal pathogens specifically enhances miR-146a in ApoE-/- mice during experimental periodontal disease. Infect Immun 2011; 79: 15971605.

32 Rodriguez A, Vigorito E, Clare S, et al. Requirement of bic/microRNA-155 for normal immune function. Science 2007; 316: 608-611.

33 Tili E, Michaille JJ, Cimino A, et al. Modulation of miR155 and miR-125b levels following lipopolysaccharide/ TNF-alpha stimulation and their possible roles in regulating the response to endotoxin shock. J Immunol 2007; 179: 5082-5089.

34 Garlet GP, Cardoso CR, Campanelli AP, Martins W Jr, Silva JS. Expression of suppressors of cytokine signaling in diseased periodontal tissues: a stop signal for disease pro- 
gression? J Periodontal Res 2006; 41:580-584.

35 Fish JE, Santoro MM, Morton SU, et al. miR-126 regulates angiogenic signaling and vascular integrity. Dev Cell 2008; 15: 272-284.

36 Takayama S, Yoshida J, Hirano H, Okada H, Murakami S. Effects of basic fibroblast growth factor on human gingival epithelial cells. J Periodontol 2002; 73: 1467-1473.

37 Takayama S, Murakami S, Nozaki T, et al. Expression of receptors for basic fibroblast growth factor on human periodontal ligament cells. J Periodontal Res 1998; 33: 315-322.

38 Murakami S, Takayama S, Kitamura M, et al. Recombinant human basic fibroblast growth factor (bFGF) stimulates periodontal regeneration in class II furcation defects created in beagle dogs. $J$ Periodontal Res 2003; 38: 97-103.

39 Bulut S, Uslu H, Ozdemir BH, Bulut OE. Expression of caspase-3, p53 and Bcl-2 in generalized aggressive periodontitis. Head Face Med 2006; 2: 17.

40 Gamonal J, Bascones A, Acevedo A, Blanco E, Silva A. Apoptosis in chronic adult periodontitis analyzed by in situ DNA breaks, electron microscopy, and immunohistochemistry. J Periodontol 2001; 72: 517-525.

41 Crotti T, Smith MD, Hirsch R, et al. Receptor activator NF kappaB ligand (RANKL) and osteoprotegerin (OPG) protein expression in periodontitis. J Periodontal Res 2003; 38: $380-387$.

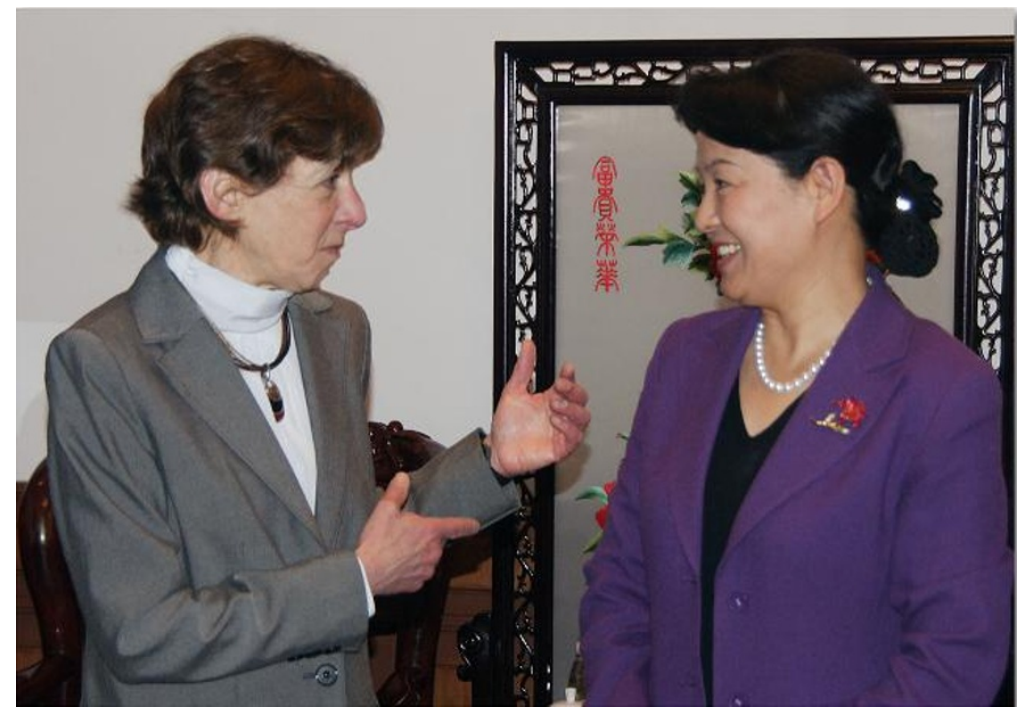

Being named as the new director of the National Institute of Dental and Craniofacial Research (NIDCR), National Institutes of Health, Professor Martha J. Somerman recently followed the NIH regulations and resigned her editorial position in International Journal of Oral Science. According to the NIDCR announcement, her appointment will be effective on August 29, 2011. Prof. Somerman has been recognized as a leading scientist and researcher in oral and maxillofacial development and regeneration, with numerous honors and awards. She is also an internationally well-known educator, serving as the Dean of the School of Dentistry, University of Washington for the past 9 years. International Journal of Oral Science congratulates Prof. Somerman for her new assignment and appreciates her generous contribution to our journal.

Photo above: Prof. Somerman (L) with Prof. Zhou Xuedong (R), the editor-in-chief of International Journal of Oral Science

\section{NIDCR news links:}

http://www.nidcr.nih.gov/NewsAndFeatures/Announcements/Somerman.htm 